\title{
Maître de langue, professeur de langue et enseignement de la musique du français $\left(\mathrm{XIX}^{\mathrm{e}}\right.$ siècle)
}

Sophie Aubin

\section{(2) OpenEdition Journals}

Édition électronique

URL : https://journals.openedition.org/dhfles/1076

DOI : $10.4000 /$ dhfles. 1076

ISSN : 2221-4038

Éditeur

Société Internationale pour l'Histoire du Français Langue Étrangère ou Seconde

Édition imprimée

Date de publication : 1 décembre 2005

Pagination : 41-57

ISSN : 0992-7654

\section{Référence électronique}

Sophie Aubin, « Maître de langue, professeur de langue et enseignement de la musique du français (XIXe siècle) ", Documents pour l'histoire du français langue étrangère ou seconde [En ligne], 35 | 2005, mis en ligne le 07 septembre 2011, consulté le 27 mai 2021. URL : http://journals.openedition.org/ dhfles/1076 ; DOI : https://doi.org/10.4000/dhfles.1076

Ce document a été généré automatiquement le 27 mai 2021.

(c) SIHFLES 


\title{
Maître de langue, professeur de langue et enseignement de la musique du français (XIX ${ }^{\mathrm{e}}$ siècle)
}

\author{
Sophie Aubin
}

\section{Introduction}

1 L'histoire de l'enseignement des sons du français langue étrangère (ou de la prononciation) a progressé d'une manière considérable. Or elle est indissociable de l'histoire de l'enseignement de la musique du français, considérée comme «moyen de communication qui consiste dans l'art de combiner des sons, de les organiser dans le temps, de les produire harmonieusement grâce au corps humain et selon des règles spécifiques » (Aubin 1996).

2 Instituer l'Histoire de l'enseignement des musiques des diverses langues vivantes, soit se placer dans le cadre global d'un enseignement musical, présente plusieurs avantages : prendre du recul par rapport à la linguistique, à la phonétique ou toute discipline connexe, tenir compte de l'interdisciplinarité qui existe entre la pédagogie musicale et la didactique des langues étrangères, inclure l'enseignement de tous les phénomènes sonores et musicaux inhérents à la communication parlée qui ont pu être enseignés, intégrer toutes les pratiques musicales individuelles des enseignants et apprenants.

Les documents d'époque qui témoignent de la réalité sonore et musicale de la classe (notes d'observation, cahiers de préparation, agendas, carnets de bord, lettres entre maîtres et élèves, descriptions et transcriptions de la musique du discours des maîtres et élèves) se font rares. E. Hammar (1998: 108) remarque que « pour la prononciation encore plus que pour le reste de l'enseignement linguistique, il nous reste extrêmement peu d'autre matière de recherche » que les manuels. Pour l'enseignement de la musique d'une langue, nous sommes encore plus démunis car une approche musicale repose en grande partie sur des particularités individuelles du maitre: sensibilité auditive, gestuelle, inspiration à un moment donné. Seuls les élèves et les collègues les plus 
proches ont pu l'attester. Dans les grammaires, manuels et dictionnaires il est surtout question de descriptions de sons et de règles de prononciation. C'est donc en confrontant mon regard historique avec des études générales sur l'histoire de l'enseignement du français, avec l'histoire particulière de l'enseignement des sons et de la prononciation du français en Europe (Italie, Suède, Espagne, Allemagne, Pays-Bas) ${ }^{1}$ et avec l'histoire de la phonétique, que je pars à la recherche du «maître et du professeur de musique du français », dans la tourmente du XIX ${ }^{e}$ siècle.

\section{Approches artistiques}

\section{L'art de parler français}

4 La présentation artistique des siècles précédents ${ }^{2}$ ne disparaît pas complètement au $\mathrm{XIX}^{\mathrm{e}}$. En 1855 paraît en Espagne le Novísimo arte teorico, práctico y sintético de la lengua francesa [...] de Delaborde. En 1867, Claude Marcel publie L'étude des langues ramenée à ses véritables principes ou l'art de penser dans une langue étrangère. En 1877 arrive L'art de la lecture de E. Legouvé (Galazzi 1997 : 167). En 1880, François Gouin suit cette tradition avec L'art d'enseigner et d'étudier les langues. On trouve même en 1892, de J. Coste un ouvrage record: Arte de hablar francés, en 8 días sin maestro ${ }^{3}$. Qu'il se rapproche de la rhétorique (L'art de bien dire ou l'art de parler de manière à persuader Littré) ou qu'il signifie méthode, art laisse toujours planer une grande difficulté, impose le respect, tout en rappelant constamment la nécessité d'avoir des aptitudes naturelles et de poursuivre un idéal esthétique.

\section{L'art d'enseigner le français}

5 L'obligation des maîtres était de dominer aussi bien l'art de parler français que celui de l'enseigner. François Gouin reprend donc l'idée d'une double action artistique (étudier et enseigner) que l'on rencontre souvent au XVIII ${ }^{e 4}$. D'emblée, les maitres de langues vivantes sont placés par les auteurs dans un courant perfectionniste proche de l'utopie. Les dons pour l'enseignement occupent une place essentielle puisque la formation était principalement autodidacte et peu favorisée par des conditions de vie modestes ou pauvres. De ce point de vue, de nombreux maitres de langues rejoignent le lot des artistes.

\section{Un art d'agrément}

6 Contrairement à l'art de parler et d'enseigner le français, le français « art d'agrément ", comme la musique, la peinture, la danse, la couture, implique un enseignement et un apprentissage aisés. Le français entre dans le cadre de la formation artistique complète d'une jeune fille "de bonne famille». Jamais les enseignements du français langue vivante étrangère et de la musique n'auront été explicitement si proches. Puisque l'objectif était le plaisir de l'écoute et de la conversation, l'étude de la musique du français devait logiquement y occuper une place prépondérante.

\section{Un art musical explicite}

7 La question ici est de savoir dans quelle mesure cet art ouvertement proclamé était de nature musicale. Rencontrer une approche artistique musicale explicite est difficile. De 
plus, celles qui se sont produites ont eu paradoxalement peu d'échos. Deux déclarations, produites à une centaine d'années d'intervalle, attirent néanmoins l'attention.

- En 1787, en Écosse, Henry Tourner avait ébauché une définition musicale de «prononciation»: «La prononciation étant la partie musicale d'une langue, ne s'acquiert qu'à partir de la bouche d'un maître capable » (cité par Wakely 1997 : 87).

9 - En 1897, aux Pays-Bas, A. G. van Hamel, professeur de philologie historique, offre un brillant éloge de la musique du français :

Tout en faisant du français une science sérieuse je ne saurais oublier qu'elle est en même temps un art, et un des plus exquis, puisque son objet est une des langues les plus artistiques qui ait jamais été parlée [...] que toute langue est une musique en même temps qu'une pensée et que si rien n'est clair comme la pensée française, il est peu de musiques aussi douces à l'oreille que le parler de France (cité par Kok Escalle 1999 : 96).

10 Van Hamel aurait donc pu écrire le «discours sur l'universalité de la musique de la langue française » qui n'a pas été écrit.

\section{Un art musical implicite}

11 Étant donné le caractère exceptionnel des approches musicales directes, où se cache la musique du français au XIX ${ }^{\mathrm{e}}$ ?

\section{- « Musique » chez Rivarol}

13 Bien que le texte De l'universalité de la langue française ne semble pas avoir eu grand succès au XIX $\mathrm{X}^{\mathrm{e}}$ siècle ${ }^{5}$, il est d'un grand intérêt car Rivarol est maître dans l'art d'évoquer les musiques de langue sans jamais les nommer directement. Cet auteur évite soigneusement les expressions "musique du français», "musique de la langue française ", au profit de "harmonie » souvent rencontrée dans les passages les plus musicaux :

La prononciation de la langue française porte l'empreinte de son caractère; elle est plus variée que celle des langues du midi, mais moins éclatante ; elle est plus douce que celle des langues du nord, parce qu'elle n'articule pas toutes ses lettres. Le son de l'e muet, toujours semblable à la dernière vibration des corps sonores, lui donne une harmonie légère qui n'est qu'à elle (1946: 78).

Or ce choix délibéré ou inconscient d'insinuer une musique en multipliant les tournures élogieuses, les adjectifs qui la caractérisent, est une tendance générale qui se retrouve dans les ouvrages destinés à l'étude et l'enseignement du français. Deux raisons supposées de cette absence contradictoire chez Rivarol sont à retenir. D'une part, c'est la langue italienne qui est "universellement» reconnue comme langue «musicale », seule capable d'épouser la "grande musique " ${ }^{6}$. D'autre part, le concept d'universalité de la musique tonale classique battait son plein, ce qui devait freiner l'usage de « musique » dans le champ de la défense de la langue française.

- Un enseignement musical fragmenté

L'histoire de l'enseignement de la prononciation démontre une grande préoccupation pour la description et l'étude de sons détachés. Ce poids contraste avec les apparitions sporadiques des phénomènes prosodiques. E. Hammar (1998:115) souligne que jusqu'à la Réforme, «les questions de l'accent, de l'intonation et de la prosodie ne sont soulevées que par intermittence ». En Flandre par exemple, il n'y a qu'un enseignant et 
auteur de grammaires françaises, Blieck, qui met en relief l'accent tonique placé sur la "syllabe dominante sur laquelle on appuie en haussant la voix " ${ }^{7}$. Ce n'est que dans les textes officiels de la fin du siècle que le rythme et la mélodie accentuent leur présence théorique en France notamment, même si leur importance est toujours secondaire par rapport aux sons : "Après que la prononciation de chaque mot aura été établie, on partagera la phrase en ses compartiments naturels, et on lui donnera son rythme $»^{8}$. produit. En 1879, pour B. Sijmons, philologue néerlandais, la prononciation renvoie à une «bonne expression orale " (d'après Kok Escalle, 1999 : 101).La définition de Littré s'étend rapidement à l'ensemble de l'expression parlée : "Manière de prononcer, de faire entendre les lettres, les syllabes, les mots, manières de dire, de débiter " . Cependant, les limites de "prononciation" sont clairement perceptibles lorsque les auteurs l'opposent à d'autres termes qui se réfèrent aux enchaînements et inflexions des sons : « prononciation et élocution $»^{10}$ (soit deux parties de la rhétorique ancienne), « accent et prononciation ${ }^{11}$.

19 Les enseignants-auteurs tournent autour de «musique » à l'aide de "prononciation », « accent ", « enchaînement ", " éloquence ", « élocution ", " articulation ", « diction " Le maître de langue française, pris sous l'angle artistico-musical, se trouve dans une situation délicate et originale. Avec son statut ambigu de maitre d'art explicite/art musical principalement implicite, il doit faire face au besoin croissant d'apprendre des variations musicales du français moderne de nature très différente (politique, sociale, culturelle, commerciale), dont la fonction est stratégique :

20 1) La musique du français mondain, spécificité des femmes, pour briller dans les salons, qui doit servir à « accueillir convenablement les étrangers » (Pellandra 1999 : 171).

21 2) La musique du français patriotique, qui va de pair avec l'époque napoléonienne et l'expansion coloniale : « (La phrase) Tous les Français doit être prononcée d'un ton plus haut que le reste. Cette émission doit porter dans les cœurs l'amour de la patrie et l'attendrissement » (C. Marazzini 1989, cité par Pellandra 1998 : 27).

22 3) La musique du français technique et commercial, spécificité des hommes, dont l'enseignement n'est pas attesté à notre connaissance.

\section{Pratiques de pédagogie musicale}

23 Malgré la domination de l'implicite musical, trois genres de pratiques plus proches de la pédagogie musicale et instrumentale que de la pédagogie des langues sont repérables. Elles ont fait largement leurs preuves en pédagogie musicale d'hier et d'aujourd'hui alors qu'elles ont été presque toutes abandonnées ou délaissées au fil des siècles en langue étrangère.

\section{Recours à l'analogie entre langue et musique}

Il s'agit d'une technique constante en pédagogie musicale ancienne et moderne (Aubin 1996) mais décroissante en pédagogie du français langue moderne : bien présentes aux $\mathrm{XVII}^{\mathrm{e}}$ et XVIII ${ }^{\mathrm{e}}$ siècles ${ }^{12}$, elles se font plus rares au XIX ${ }^{\mathrm{e}}$. En 1826 par exemple, Antonio 
Scoppa en Italie défend dans sa méthode la supériorité de la perception auditive sur la perception visuelle : "La prononciation comme la musique ne concerne que l'oreille » (cité par Minerva, 1997 : 62). P. J. Rousselot en 1899 effectue une analogie retentissante de nature instrumentale (sur laquelle je reviendrai) entre le maitre de langue et le maitre de piano: «Le maître lui [le jeune pianiste] met le doigt sur la touche qui correspond à la note demandée. Pourquoi le maître de langue ne ferait-il pas de même?» (Galazzi 1993 : 74).

\section{Utilisation d'expressions imagées}

Caractériser les sons du français en expliquant la bonne manière de les prononcer, d'une façon subjective, sensorielle et affective, peu soucieuse de rigueur scientifique, à l'aide d'images très simples pour maîtres et élèves (ce qui fera parfois sourire la phonétique moderne appliquée) est également une technique d'enseignement habituelle en pédagogie musicale. Cette pratique, fréquente aux siècles précédents ${ }^{13}$, subsiste dans la seconde moitié du XIXe. Les manuels d'Averardo Montesperelli en apportent un exemple : des sons doux, légers, délicats, prononcer le $\mathrm{U}$ « comme si on voulait siffler » (cité par Bossut, $1997: 80$ ).

\section{Mémorisation auditive et étude de morceaux choisis}

C'est dans la méthode dite "traditionnelle ", encore largement pratiquée au XIX" , que l'on trouve le plus de types d'activités appartenant à la pédagogie musicale : dictées ${ }^{14}$, lecture, écoute, répétitions, imitations, composition, mémorisation de morceaux choisis, reprise en chœur, etc. La part réservée à la mémorisation auditive, au silence, aux sonorités et à la musicalité y est nécessairement considérable afin de permettre des enregistrements naturels. La « voix haute » fait écho à la « vive voix du maître ».

Cette dose de pédagogie musicale dans le champ de l'enseignement d'une langue vivante, très variable selon les profils des maîtres, permet de considérer comme facteur déterminant l'influence sur l'enseignement des langues étrangères d'une formation musicale parallèle et complémentaire que des maîtres de langue possédaient, grâce à l'étude et la pratique inavouée (à ma connaissance) mais facilement perceptible, d'un instrument de musique. D'ailleurs, nous savons que dans les milieux sociaux où l'étude de la langue française était de règle, celle de l'étude d'un instrument de musique l'était aussi et que la musique vivante faisait partie de l'environnement sonore quotidien. Par conséquent, une part importante des maîtres et surtout des maîtresses de langue avaient ou avaient eu des contacts étroits avec la musique tonale et devaient être sensibles à la justesse du rythme, aux variations mélodiques, à l'esthétique sonore.

\section{Recherche de la perfection : la vive voix du maître}

Au XIX ${ }^{e}$ siècle, le vieux concept de la vive voix du maître, qui contraste avec la voix mourante du professeur de latin ${ }^{15}$, résiste encore avant de s'éteindre au XX $\mathrm{XX}^{\mathrm{e}}$ siècle. Il est en vigueur en Espagne (Fernández Fraile 2002) et en Italie par exemple. Dans son étude des manuels d'Averardo Montesperelli, A. Bossut remarque que «la fameuse formule “la voix du maître" est souvent mentionnée » (1997: 77). Mais qu'est-ce que cette vive voix? Qui dit «voix» dit «instrument», qui dit "vive» dit vivante et implique mouvement, musique. Cette expression, cœur de l'approche artistique, unit à la 
perfection les aspects musicaux et «instrumentaux" de l'acte de communication langagière. Voici donc notre maître doté d'un instrument de travail qui n'est autre qu'une sorte d'instrument de musique, dont la qualité doit en théorie être irréprochable. L'idéal d'un enseignement musical est poursuivi : pour être considéré comme un bon maître de langue vivante, il faut avoir une bonne voix et savoir s'en servir, à l'image du maître de chant. La recherche du bon maître de français ressemble fortement à celle du bon maître de musique.

Le maître de français doit donc dominer l'art de parler et d'enseigner, compenser les descriptions des sons jugées insuffisantes, enseigner une matière, la prononciation, reconnue comme prioritaire aux côtés de la grammaire, être doté d'une bonne vive voix sur laquelle repose le succès de l'entreprise. Ce perfectionnisme a dû être mal vécu par de nombreux maîtres dont le manque de formation et de ressources ne permettaient pas de faire face au poids de cette responsabilité. L'entretien de cette vive voix dépendait de contacts sporadiques avec des Français ou de quelques voyages. Les maitres natifs, non isolés, motivés, issus d'une classe sociale aisée, étaient privilégiés et recherchés. Étant donné les constats de bas niveau qui jalonnent les études sur l'histoire de l'enseignement de la prononciation, les «bonnes voix » étaient rares ou mal utilisées. Les " mauvais maîtres », c'est-à-dire ceux dont la prononciation n'était pas jugée satisfaisante semblent courir les rues et leur chasse était ouverte. De plus, ceux qui osaient avoir recours aux transcriptions phonétiques risquaient de ne pas jouir d'une bonne réputation: «Prétendre qu'un livre muet puisse remplacer la voix d'un bon maitre n'est que du charlatanisme $»^{16}$.

La valeur accordée à la vive voix peut être une garantie de qualité, un facteur d'inégalité entre les maîtres, un simple argument publicitaire lorsqu'elle est exploitée pour attirer la clientèle de cours privés et particuliers (Suso Lopez 1999: 188 et 189), mais aussi une source précieuse d'innovation pédagogique. Ce pouvoir stratégique, ce savoir-faire unique officiellement reconnu, confère au « maitre de prononciation » une légitimité et une liberté que le "maître de grammaire" n'a pas et que certains et surtout certaines ont su mettre à profit.

\section{Maîtresses et maîtres de musique du français}

31 Du « maître de prononciation » au «maître de musique du français » il n'y a qu'un pas, facilité par l'importance donnée à la vive voix. Il est juste d'attribuer à certains maîtres et catégories de maîtres de français le titre posthume de maître de musique du français dont nous amorçons une liste. Ils représentent un moteur de l'évolution de l'enseignement vivant des langues étrangères, la pédagogie de la prononciation ayant déjà été reconnue comme facteur déterminant de progression vers la méthode directe : "L'enseignement de la prononciation est bel et bien quelque chose de vivant, qui bouge, qui grésille de vie » (Fernández Fraile $2002: 46$ ).

\section{Bonnes et gouvernantes natives}

Pionnières en matière d'enseignement précoce des langues en situation et de « cours de conversation ", leurs méthodes dites naturelle, maternelle, féminine, sont forcément les plus musicales. De plus, ce collectif dispersé, principalement formé en moralité, travaux ménagers et manuels, renferme de multiples savoir-faire individuels. 


\section{Maîtresses de français} 1999, Pellandra 1999, Merger 2002), guidée par un allègement adapté aux filles de l'étude de la grammaire. Il s'agit bien d'un mal politique pour un bien pédagogique : «Partout le français fait partie du curriculum des jeunes filles des classes aisées avec les autres arts d'agrément [...] sans susciter aucune contestation dans la mesure où il n'entre pas en compétition avec l'enseignement des langues classiques, domaine exclusif des garçons » (Pellandra 1998 : 30). La discrimination socio-pédagogique dont elles étaient victimes (enseignement de matières "inférieures») leur donnait involontairement la marge de liberté indispensable au plein et bon usage de la vive voix. En effet, leurs classes ressemblent à des centres d'expérimentation de l'enseignement de la musique $\mathrm{du}$ français. Dans une école de filles en Italie par exemple, pendant les trois premières années de l'école primaire, les enseignantes dispensaient un enseignement strictement oral à travers «le récit d'histoires amusantes » (Pellandra 1999 : 179).

\section{Monsieur Bérard} cadre d'une étude sur l'enseignement du français dans une école de filles en Suède. Il est attesté qu'il a été exclusivement chargé en 1880/1881 de cours spécifiques " d'exercices de lecture et de conversation ». Il a joué ouvertement le rôle de maitre de production et d'expression orales, en collaboration avec ses collègues. Son statut ne semble pas subalterne mais égal à celui d'autres enseignants de français intervenant dans cette école.

\section{Pédagogues et enseignants auditifs}

Certains maîtres réagissent à la domination de l'écrit et de l'intellect en proclamant l'importance et même la priorité de la perception auditive, démontrant ainsi une sensibilité musicale forgée au contact des élèves. Il serait nécessaire d'étudier spécifiquement leur approche auditive et musicale à travers leurs œuvres et leur carrière. Outre Antonio Scoppa déjà cité pour son analogie langue-musique, deux noms peuvent être avancés.

36 - Averardo Montesperelli : étudié par A. Bossut (1997: 76-85), ce poète et maître de langues étrangères italien né en 1810 s'inscrit dans une approche artistique. A. Bossut considère qu'il s'agit de "quelqu'un qui a l'habitude de travailler avec ses oreilles ».

37 - François Gouin : selon les principes exposés dans sa méthode de 1880, l'oreille doit pleinement intervenir avant l'œil et la main : « Avant tout, il est bien entendu que l'œil et la main ne prennent possession du thème, qu'après que l'oreille l'a entièrement conquis par elle-même et transmis à l'esprit $»^{17}$.

Ce premier essai de distinction musicale tend à démontrer qu'une disciplinarisation de la musique du français ainsi qu'une orientation musicale de la profession ont été ébauchées. Ce faible mouvement, de source principalement féminine, ne pouvait guère prospérer. 


\section{Non-disciplinarisation de la musique du français}

39 C'est dans le système scolaire masculin que la mort de l'approche artistique et des "méthodes musicales", qui n'étaient qu'au stade embryonnaire, fut la plus spectaculaire. Si l'on a souvent regretté le fait que le maître de langue vivante a été transformé en professeur de langue morte (calque de la pédagogie des langues vivantes sur celle des langues mortes), que dire du sentiment des professionnels implicitement maîtres de musique du français qui n'ont pu exercer librement leur art dans leur établissement? L'entrée des langues vivantes dans les lycées publics crée trois sortes de professeurs : les professeurs de langues vivantes qui se sont mis à imiter les professeurs de langues classiques, dans l'espoir d'être mieux considérés (Puren 1988 : 75, Pellandra 1998 : 38), les professeurs de langues classiques qui exerçaient les fonctions de professeurs de français (Reinfried 1997 : 186), les professeurs désireux d'enseigner une langue vivante et musicale. Les conditions de travail de ces derniers étaient extrêmement difficiles, à tel point qu'on se demande si une liste de professeurs de musique du français, pourrait être amorcée. La vive voix qui semblait inspirer le respect dans un environnement sonore silencieux devient un drôle d'accent qui provoque l'hilarité dans le brouhaha de classes surchargées.

Avant l'institutionnalisation, le cours particulier était la formule consacrée ou le petit groupe dans le cas des jeunes filles s'exerçant aux arts d'agrément. Puis, le cours individuel s'est maintenu en dehors de l'école. Or l'enseignement exclusivement collectif de toute discipline artistique assure l'entretien d'un niveau relativement bas ou l'échec. Constater l'absence de disciplinarisation des musiques de langue au cours de l'institutionnalisation des langues vivantes, c'est toucher de plein fouet «la problématique originelle de la didactique des langues vivantes étrangères, à savoir l'adaptation de la méthode naturelle à l'enseignement scolaire » (Puren 1988 : 38). La disciplinarisation et l'institutionnalisation non seulement de la langue française mais ausside la musique du français, soit la création de cours obligatoires de musique du français, aurait exigé l'étude de l'adaptation de méthodes musicales et « instrumentales » au cadre scolaire, la prise en compte permanente des relations qui unissent une langue à sa musique et naturellement l'organisation d'une alternance entre des cours particuliers et collectifs. Une étude comparative entre la didactique des langues vivantes et la pédagogie musicale serait ici intéressante car l'institutionnalisation de l'enseignement du langage musical et des instruments de musique dans les conservatoires par exemple a permis d'atteindre un enseignement de qualité et un niveau non négligeable de démocratisation en combinant cours individuels et collectifs.

\section{Phonétique instrumentale ou musicale ?}

De toute évidence, il fallait agir pour renverser l'évolution de l'apprentissage des langues vivantes vers la fossilisation. L'orientation de la phonétique qui allait être appliquée ne pouvait être que déterminante. C'est une phonétique instrumentale, technique (considérée ici par opposition à musicale, affective) qui a pour longtemps marqué les esprits. Un bon professeur était désormais non plus celui qui savait utiliser les qualités de sa vive voix mais celui qui dominait une phonétique appliquée. À l'arrivée de la Réforme, la formation en phonétique a donc relayé le concept moribond de vive voix. Un malaise, encore perceptible à la fin $\mathrm{du} \mathrm{XX}^{\mathrm{e}}$ et souvent constaté (voir 
Aubin, sous presse), a pris racine. Le plus grand travers des phonéticiens modernes a peut-être été de promouvoir l'étude d'un instrument de sons, au lieu d'un instrument de musique. L'analogie instrumentale et non musicale avec le maitre de piano de Rousselot est donc ici très significative. Nous trouvons pourtant l'expression « musique du langage » au programme des cours de l'Alliance Française de 1899 (Galazzi 1997 : 74) mais il s'agirait plus d'un argument publicitaire (à moins que le cours de «musique du langage " n'ait été un échec) qu'une contribution à l'institution d'une matière à part entière, à la création d'une formation continue en enseignement de cette musique spécifique car l'expression ne réapparait pas les années suivantes.

Si l'on parcourt l'histoire de la phonétique de Bertil Malmberg (1991: 377-405), on remarque que dès le début du XVIII ${ }^{e}$ l'instrument de musique est très présent. Plusieurs précurseurs et pionniers de la phonétique moderne se fondent sur l'analogie entre les mécanismes de production de sons de parole et ceux d'instruments de musique : D. Dodart en 1703, A. Ferrein en 1741, Hermann von Helmholtz en 1863. La phonétique moderne est née de l'observation de sons d'instruments de musique et de leur comparaison avec les sons émis par l'appareil phonatoire humain. Cependant, Eduard Sievers (1850-1932), linguiste et professeur de philologie romane et germanique, s'écarte de l'approche strictement instrumentale, d'abord en s'appuyant sur la pédagogie du chant, puis en définissant une phonétique plus humaine et musicale car elle valorise les qualités sonores uniques de la production de tout sujet parlant et accorde une grande importance au rythme :

[...] son intérêt scientifique pendant la deuxième moitié de sa vie fut absorbée par l'analyse sonore. Il voyait dans la façon de produire les sons du langage et dans les particularités sonores et rythmiques qui s'ensuivaient une caractéristique de chaque individu, son signalement sonore. [...] La postérité n'a pas pu le suivre, même s'il y avait derrière ses idées quelques notions de base dignes d'intérêt (Malmberg 1991 : 321).

Nous nous joignons à B. Malmberg pour regretter que ces thèses n'aient pas été plus diffusées et n'aient pas reçu une plus grande adhésion. Force est de penser que si cette orientation phonétique avait eu plus d'adeptes, si Sievers et Rousselot avaient formé un duo par exemple, la première vague d'application de la phonétique moderne à la didactique du FLE aurait peut-être été teintée de sonorité et musicalité.

\section{Conclusion}

Parmi les mouvements du XIX ${ }^{e}$ siècle se trouvent donc l'abandon de l'approche artistique et la dissociation croissante entre la langue et sa musique sur le plan disciplinaire, entre la pédagogie du français et la pédagogie musicale sur le plan interdisciplinaire. L'institutionnalisation a fortement cloisonné les disciplines langue et musique. Dévoiler les maillons d'une chaîne didactico-pédagogique musicale propre à l'enseignement des langues vivantes étrangères est possible, même si elle se réduit parfois à un fil. Ce rapide regard contribue à alimenter ma thèse selon laquelle l'enseignement de la musique du français a toujours plus ou moins existé et qu'il devrait être définitivement professionnalisé et institutionnalisé. 


\section{BIBLIOGRAPHIE}

AUBIN, Sophie. 1996. La didactique de la musique du français : sa légitimité, son interdisciplinarité, thèse de doctorat, Rouen, www. Anrtheses.com.fr, no 23865 .

AUBIN, S. Sous presse. «Histoire de l'enseignement de la prononciation : de la phonétique appliquée vers une didactique musicale ", Le français : histoire, langue et culture face aux défis actuels, $\mathrm{VI}^{\mathrm{e}}$ congrès international de linguistique française, 4-6 novembre 2003, Université de Grenade.

BERRÉ, Michel, BOONE, Annie. 1999. «L'enseignement de la prononciation dans les manuels de français publiés en Flandre entre 1763 et $1856 »$. Documents 19 : 98-115.

BossuT, Annette. 1997. « La prononciation dans les manuels de langue française pour Italiens d'Averardo Montesperelli », Documents 19 : 76-85.

CAPPELLO, Maria Luisa. 1994. "L'enseignement de la prononciation du français aux XVIII ${ }^{\mathrm{e}}$ et XIX siècles en Italie », Documents $14: 144-150$.

CARUANA-DINGLI, Noël, AQUILINA, Anthony. 1999. «Évolution et caractéristiques de l'institutionnalisation du français en milieu scolaire maltais entre 1850 et 1900 », Documents 23 : 65-81.

CLAVÈRES, Marie-Hélène. 1999. « Langue et littérature. La littérature dans l'enseignement de l'anglais en France jusqu'en $1969 »$, Documents 23 : 18-64.

DESNÉ, Roland. 1998. «Rivarol : les mots d'un débat ». Le français dans le monde, Recherches et applications : Histoire de la diffusion et de l'enseignement du français dans le monde, janvier: 48-49. FERNÁNDEZ FRAILE, M M Eugenia. 2002. «L'enseignement de la prononciation du français en Espagne au XIXe siècle ", Documents $28: 33-51$.

GALAZZI, Enrica. 1993. « Machines qui apprennent à parler, machines qui parlent : un rêve technologique d'autrefois ». Études de Linguistique Appliquée 90 : 73-84.

GALAZZI, E. 1997. «Physiologie de la parole et enseignement de la prononciation fin $19^{\mathrm{e}} /$ début $20^{\mathrm{e}}$ siècles ». Documents $19: 166-183$.

HAMMAR, Elisabet. 1998. «La prononciation au temps des pédagogues ». Le français dans le monde, Recherches et applications: Histoire de la diffusion et de l'enseignement du français dans le monde, janvier : 106-117.

HAMMAR, E. 1999. «Analyse d'une année scolaire d'apprentissage du français dans une école de filles en Suède à la veille du mouvement de la Réforme ». Documents 23 : 141-167.

KOK ESCALLE, Marie-Christine. 1999. « Le français aux Pays-Bas dans la deuxième moitié du XIX ${ }^{\mathrm{e}}$ siècle ». Documents 23 : 83-107.

MALMBERG, Bertil. 1991. Histoire de la linguistique, de Sumer à Saussure. Paris : PUF.

MANDICH, Anna Maria. 1997. «Comment décrire les sons? La description phonétique dans les grammaires publiées en Italie aux $17^{\mathrm{e}}$ et $18^{\mathrm{e}}$ siècles $»$. Documents $19: 23-36$.

MERGER, Marie-France. 2002. «L'enseignement du français à Lucques de 1805 à 1814 : une expérience novatrice ». Documents $28: 71-86$.

MINERVA, Nadia. 1997. « Les sons nasaux enseignés aux Italiens aux $17^{\mathrm{e}}$ et $18^{\mathrm{e}}$ siècles ». Documents $1997: 51-64$. 
NIEBERDING, Béatrice. 1997. « Didactique de la dictée en FLE. Sur les exercices de discrimination phonétique ». Documents $19: 144-157$.

PELLANDRA, Carla. 1994. « La scolarisation d'un art d'agrément : l'enseignement du français en Italie au XIX'e siècle ». Documents 14 : 92-101.

PELLANDRA, C. 1998. «1780-1880 : De l'universalité aux résistances nationales ». Le français dans le monde, Recherches et applications: Histoire de la diffusion et de l'enseignement du français dans le monde, janvier: 24-46.

PELLANDRA, C. 1999. «L'enseignement du français aux jeunes filles italiennes dans la seconde moitié du XIXe siècle ». Documents $23: 168-184$.

PUREN, Christian. 1988. Histoire des méthodologies de l'enseignement des langues. Paris : Nathan.

REINFRIED, Marcus, 1997. « Le mouvement réformiste en Allemagne ». Documents 19 : 184-197.

RIVAROL, Antoine. 1946. De l'universalité de la langue française, Discours qui a remporté le prix à

l'académie de Berlin en 1784. Paris : Éditions des quatre vents.

SUPIOT, Alberto. 1996. « Les manuels de Français Langue Étrangère en Espagne entre 1648 et 1815. Approche bibliographique ». Documents 18 :313-328.

SUSO LÓPEZ, Javier. 1999. « La méthode naturelle d'apprendre la langue française dans la première moitié du XIX ${ }^{\mathrm{e}}$ siècle en Espagne ». Documents 23 : 185-203.

WAKELY, Robert. 1997. «Utilité des indications phonétiques dans les manuels de français publiés en Écosse et en Angleterre aux $18^{\mathrm{e}}$ et $19^{\mathrm{e}}$ siècles ». Documents 19 : 86-97.

\section{NOTES}

1. En examinant notamment les travaux du colloque de Linköping en 1996 : "Phonétique et pratiques de prononciation, L'apprentissage de la prononciation : chemin parcouru jusqu'à nos jours ", Documents 19 et ceux du colloque de Sintra en 1998: "L'enseignement de la langue et de la littérature françaises dans la seconde moitié du XIX ${ }^{\mathrm{e}}$ siècle ", Documents 23.

2. Les titres d'ouvrages contenant le mot art sont assez nombreux. Pour l'Espagne: Pedro Burgea (1672), Artepara aprenderfacilmente y en poco tiempo a leer, escrivir y hablar la LenguaFrancesa, Juan Pedro Jaron, (1688), Arte nuevamente compuesto de la Lengua Francesa, Pedro Nicolas Chantreau (1781), Arte de hablar bien francés; voir par exemple les recherches bibliographiques d'Alberto Supiot(1996).

3. Art de bien parler français en 8 jours et sans maître (voir Fernández Fraile 2002 : 48).

4. Exemples: Michele Berti, Jacques Contois, L'arte d'insegnare la lingua franzese (1734), A. N. Pluche, La mécanique des langues et l'art de les enseigner (1735).

5. «Si le mérite de Rivarol fut célébré en France, son discours ne semble pas avoir été, pour les contemporains, un succès de librairie, à en juger par le catalogue de la B.N. : deux éditions en 1784, deux autres en 1797, une en 1802 [...]. Il ne sera pas réédité avant 1889. » (Desné $1998:$ : 49).

6. À propos de la langue italienne: «la prose, composée de mots dont toutes les lettres se prononcent, et roulant toujours sur des sons pleins, se traîne avec trop de lenteur ; son éclat est monotone; l'oreille se lasse de sa douceur, et la langue de sa mollesse : ce qui peut venir de ce que chaque mot étant harmonieux en particulier, l'harmonie du tout ne vaut rien. [...] Malgré tout cela [...] la plus mélodieuse des langues s'est unie à la musique des anges, et cette alliance lui assure un empire éternel. » (22-24).

7. Cité par Berré / Boone, 1999 : 107. 
8. Arrêté du 28 janvier 1890 pour l'enseignement de l'anglais en France (Clavères 1999 : 43).

9. Aujourd'hui, les diverses acceptions sont plus clairement définies et celle qui nous concerne est limitée au domaine précis de l'articulation des sons : «Manière dont les sons du langage sont articulés, dont un mot est prononcé. Prononcer : Articuler d'une certaine manière (les sons du langage) », Le Robert, Dictionnaire pratique de la langue française, 2002.

10. Extrait du programme d'évaluation pour passer le certificat d'aptitude à l'enseignement primaire, loi de 1857, Pays-Bas, cité par Kok Escalle, 1999 : 90.

11. Extrait du rapport du commissaire P. J. Keenan à l'issue d'observations de classes à Malte en 1880, cité par Caruana-Dingli / Aquilina, $1999: 71$.

12. 1687, début de la grammaire de P.P. Billet : « Les lettres sont, par rapport aux langues, ce que les notes sont pour la Musique. [...] Sans la connaissance parfaite des notes, personne ne peut se dire Musicien ; sans la science des lettres, personne ne peut se vanter de savoir avec élégance une langue » (cité par Fischer, 1997 : 38). 1698, Grammaire de Richany-Dall'Oglio : « Il faut donc que la prononciation soit douce, naturelle, et sans cadence, ce qui est typique du chant» (cité par Mandich, 1997 : 25).

13. XVII ${ }^{\mathrm{e}}$, description des nasales : "Des expressions imagées reviennent qui indiquent qu'on coupe, qu'on arrête, qu'on freine le son, un son qui reste presque suspendu en l'air » (Minerva, 1997 : 58). 1772, Goudar : la prononciation du S ne doit pas «blesser l'oreille par sa dureté », cité par Mandich, $1997: 33$.

14. Béatrice Nieberding affirme que «La dictée est d'abord un exercice d'écoute et d'apprentissage de l'écoute ». Elle dévoile un enseignement de la prononciation par la dictée (1997 : 144).

15. Crise de la didactique du latin parlé, Puren 1988 : 24-33.

16. Portugal, 1851, Miguel de Bourdiec, traducteur, cité par Salema, $1997: 119$.

17. F. Gouin, Exposé d'une nouvelle méthode linguistique. L'art d'enseigner et d'étudier les langues, cité par Kok Escalle, 1999 : 90.

\section{RÉSUMÉS}

Toute langue est si liée à sa musique qu'il faut logiquement envisager la recherche du maître de musique de langue et l'évolution vers le professorat. Le bon maître de langue devait être un artiste de qualité : les approches musicales plus ou moins feutrées fusent au XVIII ${ }^{\mathrm{e}}$ et perdurent au XIX ${ }^{\mathrm{e}}$. Les domaines de la Langue et de la Musique se croisent, se rejoignent. Des portraits de maîtres et maîtresses de musique du français s'esquissent. Cependant, l'exclusion de ce courant artistico-musical du processus d'institutionnalisation, l'application d'une phonétique plus instrumentale que musicale, affective et humaine ont déterminé le sort de l'enseignement des musiques de langue, fondé sur la non-disciplinarisation et le cloisonnement des matières linguistiques et musicales. Malgré la Réforme et de profondes racines historiques, le professeur de musique du français n'a jamais reçu de base institutionnelle.

Every language is so closely related to its music that it is obviously necessary to face the research of the language music teacher and the evolution towards teaching. A good language teacher should be an artist of quality: the musical approaches, more or less silent, were developed during the $18^{\text {th }}$ century and continued during the $19^{\text {th }}$. The fields of Language and Music meet, link up. The portraits of the teachers of French music can be sketched. Nevertheless, the exclusion of this 
artistic-musical current from the institutionalisation process, the application of a phonetic that is more instrument than musical, affective or human have determined the kind of teaching of the music of languages, based upon the non-disciplinarisation and the compartmentalization of language and music subjects. Despite the Reform and the deep historical roots, the teacher of music of French has never had an institutional basis.

\section{INDEX}

Mots-clés : art, Français Langue Etrangère, musique, pédagogie musicale, phonétique, prononciation

Keywords : art, French as a foreign language, music, musical pedagogy, phonetics, pronunciation

\section{AUTEUR}

\section{SOPHIE AUBIN}

Institut français de Valence 